\title{
Papel de los polimorfismos -238 y -308 del promotor del factor de necrosis tumoral alfa en la patogenia y respuesta al tratamiento anti- factor de necrosis tumoral alfa en artritis reumatoide
}

\author{
Carolina Llanos $\mathbf{M}^{1}$, Lilian Soto $S^{1}$, Francisca Sabugo $S^{1}, M$ \\ José Bastías $\mathrm{Ch}^{1}$, Lorena Salazar $\mathrm{A}^{2 \mathrm{2a}}$, Juan C Aguillón $\mathrm{G}^{2 \mathrm{~b}}$, \\ Miguel Cuchacovich $\mathrm{T}^{1}$. \\ The influence of -238 and -308 TNF $\alpha$ \\ polymorphisms on the pathogenesis \\ and response to treatment in \\ rheumatoid arthritis
}

Rheumatoid arthritis (RA) is a systemic autoimmune disease that affects $0.8 \%$ of the world population, it affects the synovial membrane of joints and the clinical presentation encompasses a wide spectrum, ranging from a mild to a severe and erosive disease that causes joint and cartilage destruction which finally provokes irreversible structural damage and patient disability. In the last years, there have been important advances in the pathogenesis of this disease, the efforts have been concentrated on pro-inflammatory cytokines such as tumor necrosis factor alpha (TNF $\alpha$ ). This protein guides numerous events in the synovial and systemic inflammatory process and is encoded in the Major Histocompability Complex (MHC), one of the most polymorphic of the genome. Polymorphisms affecting the TNF $\alpha$ gene and its regulatory regions are associated with RA prevalence and course. There is a possible association between these polymorphisms and the clinical response to the use of monoclonal antibodies anti-TNF $\alpha$. The possibility that the determination of genotypes -238 and -308 may have prognostic and therapeutic consequences is debated nowadays (Rev Méd Chile 2005; 133: 1089-95).

(Key Words: Arthritis, rheumatoid; Genotype; Tumor necrosis factor-alpha)

Recibido el 3 de agosto, 2004. Aceptado el 14 de marzo, 2005.

Financiamiento proyectos: FONDECYT 2004 № 1040439 y N 1040860; HCUCH 2002 N 037/ 02.

${ }^{1}$ Sección de Reumatología, Departamento de Medicina, Hospital Clínico Universidad de Chile.

2Programa Disciplinario Inmunología; ICBM; Facultad de Medicina, Universidad de Chile.

aBioquímico; Tesista doctorado Ciencias Biomédicas.

bioquímico; PhD Ciencias

Correspondencia a: Dr. Miguel Cuchacovich T. Santos

Dumont 999; Sección Reumatología HCUCH. Fono:

6788236. E mail: mcuchaco@ns.hospital.uchile.cl 
L a artritis reumatoide (AR) es una enfermedad sistémica autoinmune que afecta a aproximadamente $0,8 \%$ de la población mundial y se caracteriza por la inflamación crónica de las articulaciones sinoviales, llevando a la destrucción del cartílago y el hueso articular. Lo anterior se traduce, en la práctica clínica, en deformidades articulares características de la enfermedad, pero, lo más importante, en discapacidad, deterioro en la calidad de vida y disminución en la expectativa de vida de los pacientes. La etiología y la patogénesis de la enfermedad son complejas y no han sido completamente dilucidadas ${ }^{1,2}$. Hay consenso que existe una contribución genética al desarrollo de la AR, dada en gran medida por la presencia de ciertos genes HLA-DRB1, sin embargo, estos genes no explican del todo el desarrollo de la enfermedad, por lo que se ha planteado que sería más bien poligénica.

A medida que se ha ido conociendo mejor la fisiopatología y los mecanismos inflamatorios, que comandan el daño articular en la $\mathrm{AR}$, se ha podido estudiar también algunos genes candidatos, que contribuirían a explicar la predisposición genética de esta enfermedad. Se ha descrito que los linfocitos T CD4+, antigénicamente activados, son los mediadores centrales de la inflamación y daño articular. Estos linfocitos, que secretan interferón gamma (IFN- $\gamma$ ) e IL-17, inducen la secreción por parte de los monocitos/macrófagos de interleuquina-1 (IL-1), IL-6 y factor de necrosis tumoral alfa $(\mathrm{TNF} \alpha)$. La sobreexpresión de TNF $\alpha$ e IL-1 parecerían ser vitales, tanto en el proceso inflamatorio como en el daño articular ${ }^{3,4}$. En este sentido, se ha visto que en el líquido y tejido sinovial de pacientes con AR existen concentraciones elevadas de $\mathrm{TNF}^{2,5,6}$ y que ratones transgénicos que sobreexpresan esta citoquina, desarrollan una enfermedad similar ${ }^{5}$. Sumado a lo anterior, los resultados del tratamiento con anticuerpos monoclonales anti-TNF $\alpha$ en pacientes portadores de AR, han demostrado un beneficio clínico significativo, permitiendo incluso detener la progresión de la enfermedad en los pacientes respondedores ${ }^{7,8}$. Por esto, la atención se ha centrado fundamentalmente en citoquinas como TNFo e IL-1.

$\mathrm{El} \mathrm{TNF} \alpha$ es un homotrímero de $51 \mathrm{kD}$ sintetizado como proteína de membrana no glicosilada, principalmente en monocitos activados, aunque linfocitos T activados, células natural killers (NK) y mastocitos también lo sintetizan ${ }^{9}$. Un fragmento de cada subunidad es posteriormente degradado y el TNF $\alpha$ es entonces secretado para unirse a dos tipos de receptores de 55 y $75 \mathrm{kD}$ llamados tipo I y tipo II, respectivamente, y que están presentes en una gran variedad de células estudiadas. Dentro de las acciones biológicas del TNF $\alpha$, destaca su rol en la respuesta de fase aguda, participando en el reclutamiento y activación de neutrófilos y monocitos ${ }^{9,10}$. El gen que codifica para el TNFa se encuentra ubicado en el brazo corto del cromosoma 6 en el Complejo Mayor de Histocompatibilidad (MHC), en el locus TNF donde también se ubican los genes de la linfotoxina (LT) $\alpha$ y LTß, aproximadamente a $1.000 \mathrm{~kb}$ de los genes HLA-DR (Figura 1$)^{10}$.

En los últimos años, se han descrito polimorfismos en el gen del TNF $\alpha$ y sus regiones reguladoras. Estos polimorfismos serían de dos tipos, de un solo nucleótido (SNP) y los de las regiones microsatélites. Se han descrito numerosos SNPs ${ }^{5,11}$ distribuidos tanto en la zona codificante como en intrones, en el extremo $5^{\prime}$ de la región no traducida (UTR) y en la región 5' regulatoria del gen (Figura 2). Estos polimorfismos podrían afectar la producción del $\mathrm{TNF} \alpha$, generando un desbalance en el equilibrio de citoquinas y favoreciendo de esta manera estados patológicos. Se sabe, por ejemplo, que el SNP que afecta a la posición -308 del promotor del gen de $\mathrm{TNF} \alpha$, se asocia a un fenotipo productor de mayores niveles de $\mathrm{TNF}^{5,10}$. Existen también estudios, que han asociado algunos de estos polimorfismos con una mayor susceptibilidad a desarrollar AR en distintos grupos de pacientes, de acuerdo al origen étnico de la población estudiada ${ }^{12-14}$.

De los polimorfismos descritos para TNF $\alpha$, nos interesan los que afectan a la posición nucleotídica -308 y a la posición -238 (ambos son transición guanina/adenina) de la región promotora del gen.

La variación nucleotídica en la posición -308 define los genotipos TNF1 $(-308 * G)$ y TNF2 $(-308 * A)^{6}$ y se manifiesta en que los monocitos provenientes de sangre periférica de pacientes portadores del alelo TNF2, estimulados con LPS in vitro, presentan una mayor producción de la citoquina que los que llevan el alelo $\mathrm{TNF}^{10}$. Estudios realizados por nuestro grupo, en población chilena, apuntan a una mayor presencia del alelo TNF2 en los pacientes portadores de AR con 


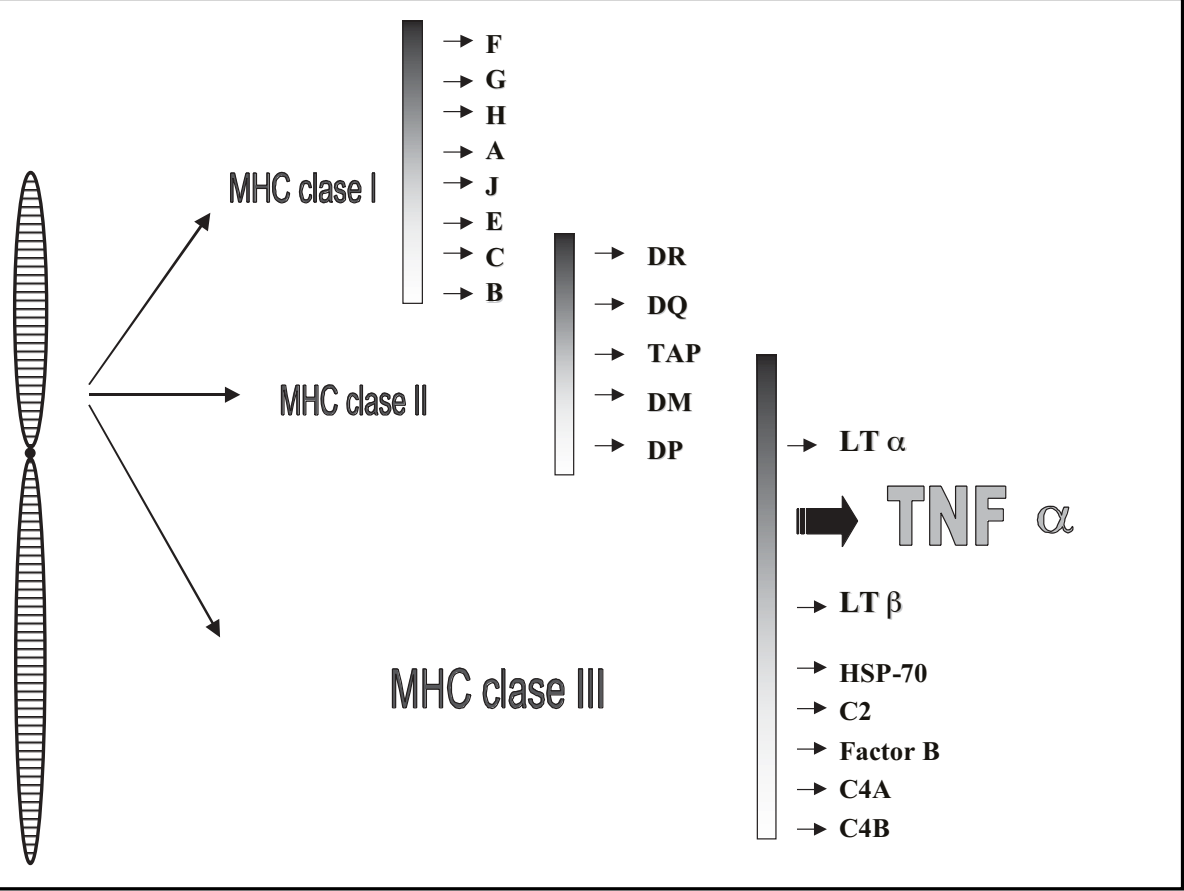

Figura 1. Localización y organización del gen de TNFa. Ubicado en el MHC clase III, en el brazo corto del cromosoma 6 humano, próximo a los genes de la LTa y LTß.

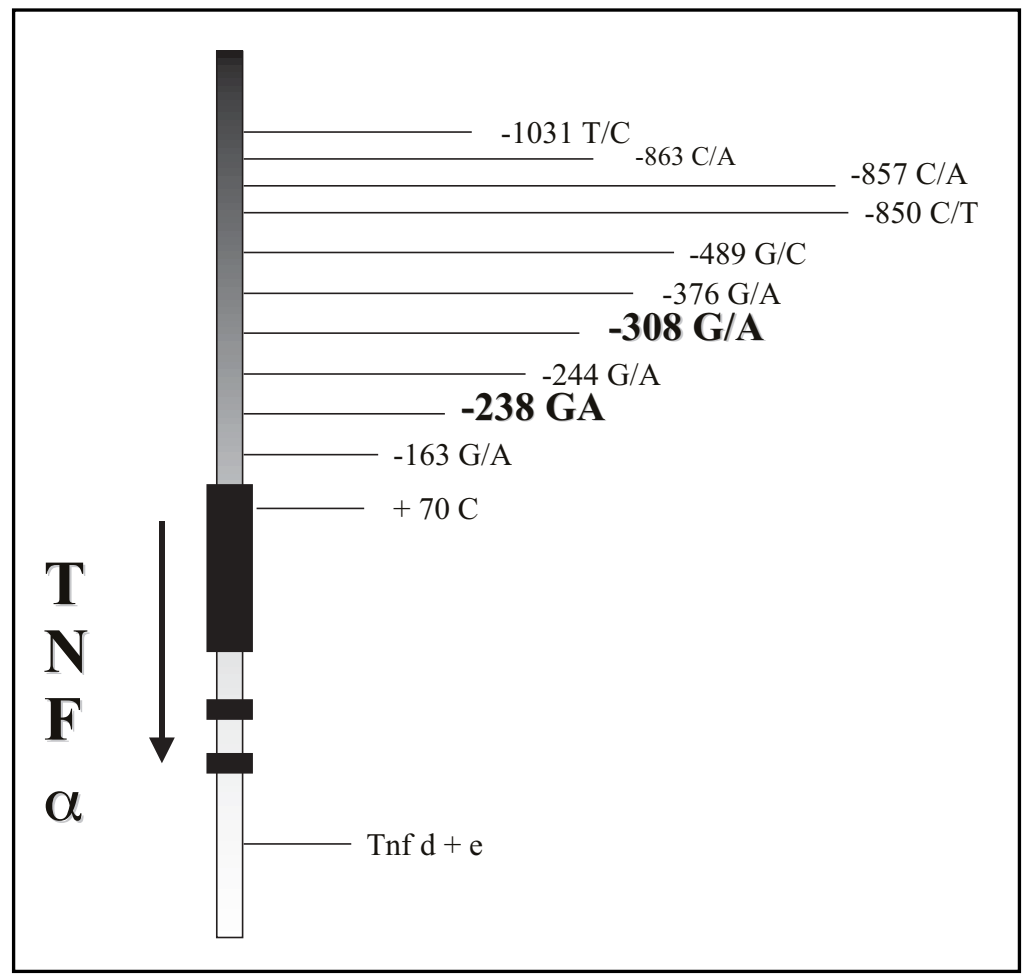

Figura 2. Se señalan en el mapa genético algunos de los polimorfismos descritos de TNFa: los microsatélites (CT)n TNF d y e, la inserción de una $\mathrm{C}$ en la posición +70 , las transiciones G/A en las posiciones -163, $238,-244,-274,-308$ y $-376, \mathrm{C} /$ $\mathrm{T}$ en las posiciones -850 y -863, $\mathrm{T} / \mathrm{C}$ en la posición -1031 y las transversiones $\mathrm{G} / \mathrm{C}$ y $\mathrm{C} / \mathrm{A}$ en las posiciones -489 y -863 , respectivamente. 
respecto al grupo control, aunque esta diferencia no alcanzó significación estadística ${ }^{13}$. Grupos orientales han descrito más bien un rol protector para el desarrollo de AR del polimorfismo -308 $\mathrm{A}^{14}$, lo que se explica probablemente por la participación de genes relacionados con las características étnicas de los pacientes estudiados. Por lo mismo, es importante destacar que, a pesar de no haber encontrado diferencias significativas entre los pacientes con AR y los controles, el perfil de distribución de frecuencias de los SNPs de TNF -308 en Chile difiere significativamente de los de poblaciones europeas, incluida la española ${ }^{13}$. Esto es concordante con la asociación descrita sólo en pacientes chilenos entre genes HLA-DR9 y $\mathrm{AR}^{15}$.

En cuanto al SNP -238, de las tres posibles combinaciones en los dos cromosomas, el GG y el GA son los más comunes. Hasta el momento no se ha detectado en las poblaciones estudiadas una mayor presencia de alguno de estos genotipos en los pacientes portadores de AR versus controles sanos $^{5}$, aun cuando existen informes de una asociación con la evolución de la enfermedad, como veremos más adelante. No hay por ahora resultados publicados en población chilena que relacionen la presencia del SNP -238 y AR, sin embargo, existen estudios en curso en nuestro grupo (proyecto Fondecyt $\mathrm{N}^{\circ} 1040439$ y proyecto Hospital Clínico Universidad de Chile 2002).

La AR es una enfermedad heterogénea, con un espectro de severidad clínica que va desde la artritis leve hasta una forma invalidante, que se caracteriza por tener altos títulos de factor reumatoide y compromiso vital de otros órganos. La mayor parte de la destrucción articular ocurre durante los primeros años de evolución de la enfermedad. Existen diversas condiciones clínicas, demográficas y genéticas que se han asociado con una evolución más severa de la AR. Dentro de estas últimas se ha investigado la asociación de los polimorfismos de TNF $\alpha$ con la severidad de la AR. Estudios realizados en España han demostrado una asociación independiente del polimorfismo microsatélite de TNFa2 con una enfermedad más erosiva. Por otro lado, el genotipo -238 GG se asocia también a una forma severa de la enfermedad, mientras que los genotipos -238 GA y +498 GG se asociarían con una menor progresión de la $\mathrm{AR}^{16,17}$. En cuanto al genotipo $-308 \mathrm{GA}$, se ha visto que en pacientes de origen turco portadores de artritis reumatoide juvenil, este polimorfismo se asocia significativamente a mal pronóstico, sin embargo, el mismo grupo no pudo confirmar estos resultados en pacientes de origen checo $^{18}$, reafirmando la importancia del origen de las poblaciones estudiadas al interpretar los resultados. Recientemente fue publicado un estudio realizado en población inglesa con AR temprana, que incluyó pacientes que consultaron por poliartritis inflamatoria en policlínicos de atención primaria. El diagnóstico definitivo de AR fue validado retrospectivamente de acuerdo a los criterios del American College of Rheumatology (ACR) a los 5 años de seguimiento. En este estudio no se demostró diferencias entre la presencia de los polimorfismos GA versus GG para las posiciones 308 y -238 y la evolución de la artritis ${ }^{19}$.

Definir la participación de los SNPs descritos y de otros genes en la etiopatogenia de la AR puede tener a futuro implicancias pronósticas y terapéuticas. Si bien es cierto que los tratamientos usados en la actualidad han logrado modificar el curso de esta patología, disminuyendo la progresión de las erosiones, aún existe un grupo importante de pacientes que se comportan como no respondedores a tratamiento convencional. En estos últimos, la terapia con anticuerpos monoclonales anti-TNF $\alpha$ logra resultados satisfactorios sólo en el $70 \%$ de los $\operatorname{casos}^{20}$. Existe, por lo tanto la posibilidad que diferencias en el perfil de expresión de citoquinas, en particular $\mathrm{TNF} \alpha$, sean determinantes en la respuesta a tratamiento con bloqueadores para TNF $\alpha$. Resultados preliminares muestran que el genotipo GG para la posición -238 se asocia a una respuesta deficiente frente al tratamiento con un anticuerpo monoclonal quimérico anti-TNF $\alpha$ denominado infliximab ${ }^{21,22}$. Por otro lado, publicaciones recientes sugieren que los pacientes portadores del genotipo -308 GG presentan una mejor respuesta al tratamiento con infliximab que los pacientes con el genotipo -308 $\mathrm{GA}^{23}$. La experiencia publicada recientemente por nuestro grupo, no muestra diferencias en la respuesta a infliximab entre las variantes alélicas GG y GA del genotipo -308 en 20 pacientes estudiados, sin embargo se encontró una correlación positiva entre los criterios de mejoría ACR y los niveles circulantes de $\mathrm{TNF} \alpha$ en los pacientes portadores del alelo $\mathrm{GA}^{24}$. Actualmente estamos realizando un estudio clínico multicéntrico para 
evaluar la respuesta al anticuerpo monoclonal recombinante humano anti-TNFo adalimumab y su correlación con la presencia de ambos polimorfismos (-238 y -308) en un grupo de 90 pacientes chilenos.

Como dijimos al inicio, los efectos biológicos del TNF $\alpha$ son mediados por la unión de esta citoquina fundamentalmente a dos tipos de receptores, el receptor de TNF tipo I p55/60 (TNFR I) y el receptor de TNF tipo II p75/80 (TNFR II) ${ }^{25}$. Ambos receptores se expresan en la membrana sinovial y pannus de las articulaciones de los pacientes con $\mathrm{AR}$, los que a su vez, presentan mayores niveles circulantes de la forma soluble de estas proteínas al ser comparados con pacientes portadores de artrosis y otras artritis inflamatorias $^{26}$. Se han descrito SNPs para ambos tipos de receptores, ubicados en la región promotora, en intrones y en exones. Se ha intentado buscar la asociación entre algunos de estos SNPs y la presencia y evolución de la AR. En el caso de TNFR I se han realizado estudios en poblaciones de origen danés, inglés y afroamericano, sin encontrar asociación entre los SNPs estudiados y el desarrollo de $\mathrm{AR}^{26}$. Los resultados obtenidos por grupos franceses e ingleses, sin embargo, han mostrado una relación entre el genotipo $196 \mathrm{R} / \mathrm{R}$ del gen de TNFR II y formas familiares de la enfermedad ${ }^{27}$. El mismo grupo describió la existencia de una asociación entre el genotipo 196 M/ $\mathrm{R}$ del gen de TNFR II y una forma de evolución más severa de la AR en términos del impacto en la capacidad funcional de los pacientes ${ }^{26}$.

A pesar del gran interés que han despertado los genes relacionados con las acciones de TNF $\alpha$ en el estudio de la fisiopatología de la $\mathrm{AR}$, los resultados de estos trabajos no son concluyentes y, en muchas oportunidades, controversiales. Por otro lado, no hay que olvidar que la asociación más fuerte entre la enfermedad y un factor genético está dada por la presencia de ciertos genes HLA-DRB1 en la región MHC clase II. Esta asociación está descrita tanto en términos de susceptibilidad a la enfermedad, como de severidad y estaría determinada en última instancia por una secuencia aminoacídica específica denominada «epitopo compartido» (EC) comprendida entre las posiciones 70 y 74 de la tercera región hipervariable de la molécula ${ }^{28}$. Los genes MHC clase II se ubican próximos al locus TNF, por lo tanto existe la posibilidad de interacción entre estos genes. Es así como en la región MHC se ha descrito desequilibrio de ligamiento (DL) entre distancias de hasta $1 \mathrm{Mb}$, con la formación de haplotipos distintos para cada alelo portador del $\mathrm{EC}^{29}$. Se ha visto, por ejemplo, que los polimorfismos de la región microsatélite TNFa11 y TNFa6 se asocian a formas severas de la enfermedad a través de su interacción con el EC $\mathrm{EC}^{30,31}$. También se ha descrito la asociación de algunos haplotipos formados por alelos de ambos locus con la respuesta a inhibidores de TNF $\alpha$. En el caso de etanercept (proteína de fusión del receptor de $\mathrm{TNF} \alpha$ ) ciertos haplotipos que incluyen alelos HLA-DRB1 y de la región TNF-LT $\alpha$ se asocian a una mejor respuesta a esta droga, dicha asociación no estaría dada por ninguno de los alelos en particular, sino por el haplotipo completo ${ }^{32}$.

La evidencia a favor de un rol para los polimorfismos del gen de TNF $\alpha$ en la susceptibilidad y pronóstico de la AR es aún controversial y los resultados deben ser interpretados con cautela, sin embargo representa un gran campo para la investigación futura en reumatología y otras disciplinas que incluyen enfermedades de etiología poligénica.

Lograr identificar factores indicadores de mal pronóstico puede permitir, por ejemplo, el uso de un tratamiento más agresivo en etapas precoces de la evolución de la AR en este grupo de pacientes. En esta área, los marcadores genéticos resultan particularmente atractivos, ya que pueden ser identificados al momento del diagnóstico de manera fácil y son constantes en el tiempo. Por otro lado, el identificar un grupo que presente mejor respuesta clínica, tanto a tratamiento convencional como a terapia biológica permitirá optimizar la utilización de los recursos disponibles. El uso racional y precoz de estas terapias podría prevenir el desarrollo de erosiones y de daño estructural irreversible que lleva finalmente a la discapacidad y deterioro en la calidad de vida para los pacientes. Por último, vale la pena destacar que la heterogeneidad de los resultados expuestos enfatizan la importancia de tener datos de nuestra población de pacientes. 


\section{REFERENCIAS}

1. Weyand C, Goronzy J. The molecular basis of rheumatoid arthritis. J Mol Med 1997; 75: 772-85.

2. Feldman M. Pathogenesis of arthritis: recent research progress. Nat Immunol 2001; 2: 771-3.

3. Brennan FM, Maini RN, Feldman M. TNFa: a pivotal role in rheumatoid arthritis. Br J Rheumatol 1992; 31: 293-8.

4. Smolen JS, Steiner G. Therapeutic strategies for rheumatoid arthritis. Nat Rev Drug Discov 2003; 2: 473-88.

5. VERWIJ C. Tumor necrosis factor gene polymorphisms as severity markers in rheumatoid arthritis. Ann Rheum Dis 1999; 58(Supl I): 120-6.

6. Vinasco J, Beraún Y, Nieto A, Fraile A, Mataran L, Pareja E et al. Polymorphisms at the TNF loci in meumatoid arthritis. Tissue Antigens 1997; 49: 74-8.

7. Lipsky P, Van Der Heidje D, St Ciair W, Furst D, BreEdveld F, KaLden J et al. Infliximab and methrotrexate in the treatment of rheumatoid arthritis. N Engl J Med 2000; 343: 1594-602.

8. Moreland L, Baumgartner S, Schiff M, Tindall E, Fieischmann R, Weaver A et al. Treatment of rheumatoid arthritis with a recombinant human tumor necrosis factor receptor (p75)-Fc fusion protein. N Engl J Med 1997; 337: 141-7.

9. ABbas A, LCHTMAn A, Pober J. Inmunología celular y molecular. Cuarta Edición 2000; capítulo 11: 248-58.

10. FIELD M. Tumor necrosis factor polymorphisms in rheumatic diseases. Q J Med 2001; 94: 237-46.

11. Uglaloro aM, Turbay D, Pesavento PA, Delgado JC, MckenZie FE, GribBen JG et aL. Identification of three new single nucleotide polymorphisms in the human tumor necrosis factor-alpha gene promoter. Tissue Antigens 1998; 52: 359-67.

12. Vinasco J, Beraún $Y$, Nieto A, Fraile A, Mataran L, Pareja E et al. Polymorphisms at the TNF loci in rheumatoid arthritis. Tissue Antigens 1997; 49: 748.

13. Cuenca J, Cuchacovich M, Ferreira L, Pérez C, Aguirre A, Schiattino I et al. The -308 polymorphism in the tumor necrosis factor gene promoter region and ex-vivo lipopolysaccharide-induced TNF expression and cytotoxic activity in Chilean patients with rheumatoid arthritis. Rheumatology 2003; 42: 308-13.

14. Yen J, Chen CH, Tsai W, Lin CH, Ou T, Wu CC et AL. Tumor necrosis factor promoter polymorphis- ms in patients with rheumatoid arthritis in Taiwan. J Rheumatol 2001; 28: 1788-92.

15. González A, Nicovani S, Massardo L, Aguirre V, Cervilia V, Lanchbury JS et al. Influence of the HLA-DRß shared epitope on susceptibility to and clinical expression of rheumatoid arthritis in Chilean patients. Ann Rheum Dis 1997; 56: 191-3.

16. Brinkman B, Huizinga T, Kurban S, Van Der Velde G, Schreuder TH, HAZes J et al. Tumor necrosis factor $\alpha$ genes polymorphisms in rheumatoid arthritis: Association with susceptibility to, or severity of, disease? Br J Rheumatol 1997; 36: 516-21.

17. Fabris M, Di Poi E, D'elia A, Damante G, Sinigagla L, Ferraccioli G. Tumor necrosis factor $\alpha$ genes polymorphism in severe and mild-moderate rheumatoid arthritis. J Rheumatol 2002; 29: 29-33.

18. Ozen S, Alukasifoglu A, Bakkaloglu A, Duzova A, Jarosova $K$, Nemcova D et aL. Tumor necrosis factor $\alpha \mathrm{G} \rightarrow \mathrm{A}-238$ and $\mathrm{G} \rightarrow$ A -308 polymorphisms in juvenile idiopathic arthritis. Rheumatology 2002; 41: 223-7.

19. Barton A, Platt H, Salway F, Symmons D, Barrett E, Bukhari M et al. Polymorphisms in the tumour necrosis factor gene are not associated with severity of inflammatory polyarthritis. Ann Rheum Dis 2004; 63: 280-4.

20. Feldmann M, MainI RN. Anti-TNF alpha therapy of rheumatoid arthritis: what have we learned? Annu Rev Immunol 2001; 19: 163-96.

21. Fabris M, Di Poi E, Sacco S, Damante G, Sinigalia L, FERRACCIOLI G. TNF $\alpha$ gene polymorphisms in rheumatoid arthritis patients treated with antiTNF $\alpha$ agents: preliminary results. Reumatismo 2002; 34: 19-26.

22. Cavaleiro J, Fonseca J, Sobral M, Mourao A, Cruz M, Carvalho T et al. Polymorphism at position 238 of the tumor necrosis factor alpha and rheumatoid arthritis: Prognosis and pharmacogenetics-interim analysis. Ann Rheum Dis 2004; 63: SI: 177.

23. Mugnier B, Balandraud N, Darque A, Roudier CH, Roudier J, Reviron D. Polymorphism at position 308 of the tumor necrosis factor $\alpha$ gene influences outcome of infliximab therapy in rheumatoid arthritis. Arthritis Rheum 2003; 48: 1849-52.

24. Cuchacovich M, Ferreira L, Soto L, Auste M, Gatica $\mathrm{H}$, CRuZAT A ET AL. TNF $\alpha$ levels and influence of -308 TNF $\alpha$ promoter polymorphism on the responsiveness to infliximab in patients with rheumatoid arthritis. Scand J Rheumatol 2004; 33: 228-32. 
25. Constantin A, Dieudé P, Lauwers-Cances V, Jamard B, Mazieres B, Cambon-Thomsen A et al. Tumor necrosis factor receptor II gene polymorphism and severity of rheumatoid arthritis. Arthritis Rheum 2004; 50: 742-47.

26. Dieudé P, Osorio J, Petit-Texeira E, Moreno S, Garnier S, Cailleau-Moindrault S et al. A TNFRI genotype with a protective role in familial rheumatoid arthritis. Arthritis Rheum 2004; 50: 413-19.

27. Dieudé P, Petit-Texeira E, Caileau-Moindrault S, Osorio J, Pierlot C, Martínez M et al. Association between tumor necrosis factor receptor II and familial, but not esporadic, rheumatoid arthritis: evidence for genetic heterogeneity. Arthritis Rheum 2002; 46: 2039-44.

28. Gorman J, Criswell L. The shared epitope and severity of rheumatoid arthritis. Rheum Dis Clin North Am 2002; 28: 59-78.
29. Newton J, Harney S, Timms A, Sims A, Rockett K, DARKE C ET AL. Dissection of class III major histocompatibility complex haplotypes associated with rheumatoid arthritis. Arthritis Rheum 2004; 50: 2122-29.

30. Mattey D, Hassell A, Dawes P, Ower W, Hajeer A. Interaction between tumor necrosis factor microsatellite polymorphisms and the HLA-DRB1 shared epitope in rheumatoid arthritis. Arthritis Rheum 1999; 42: 2698-704.

31. Mu H, Chen J, Jiang Y, King M, Thomson G, Criswell L Tumor necrosis factor a microsatellite polymorphism is associated with rheumatoid arthritis severity through an interaction with the HLA-DRB1 shared epitope. Arthritis Rheum 1999; 42: 438-42.

32. Criswell L, Lum R, Turner K, Woehl B, Zhu Y, Wang $\mathrm{J}$ ET AL. The influence of genetic variation in the HLA-DRB1 and LTA-TNF regions on the response to treatment of early rheumatoid arthritis with methotrexate or etanercept. Arthritis Rheum 2004; 40: 2750-56. 